\title{
Mechanical Fingerprint of Senescence in Endothelial Cells
}

\author{
Nafsika Chala, Silvia Moimas, Costanza Giampietro, Xinyu Zhang, Tomaso Zambelli, Vasileios Exarchos, \\ Timo Z. Nazari-Shafti, Dimos Poulikakos, * and Aldo Ferrari*
}

Cite This: Nano Lett. 2021, 21, 4911-4920

Read Online

ACCESS

山lll Metrics \& More

回国 Article Recommendations

S1 Supporting Information

ABSTRACT: Endothelial senescence entails alterations of the healthy cell phenotype, which accumulate over time and contribute to cardiovascular disease. Mechanical aspects regulating cell adhesion, force generation, and the response to flow contribute to the senescence-associated drift; however, they remain largely unexplored. Here, we exploit force microscopy to resolve variations of the cell anchoring to the substrate and the tractions generated upon aging in the nanonewton $(\mathrm{nN})$ range. Senescent endothelial cells display a multifold increase in the levels of basal adhesion and force generation supported by mature and strong focal adhesions. The enhanced mechanical interaction with the substrate yields static endothelial monolayers that polarize in response to flow
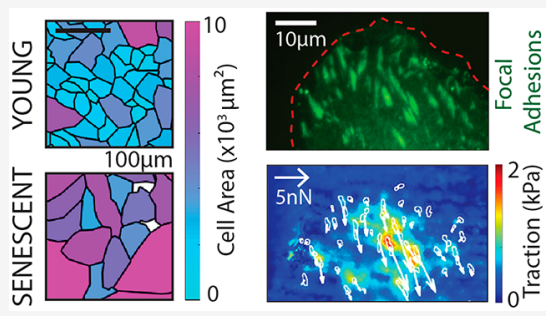
but fail the process of coordinated cell shape remodeling and reorientation. The emerging picture indicates that senescence reinforces the local cell interaction with the substrate and may therefore prevent endothelial denudation; however, it compromises the ability to functionally adapt to the local hemodynamic conditions.

KEYWORDS: endothelial cell, senescence, wall shear stress, traction force microscopy, FluidFM

$\mathrm{E}$ ndothelia are connected monolayers of endothelial cells (ECs) lining the luminal surface of blood and lymphatic vessels. ${ }^{1}$ The young and healthy endothelium is a dynamic tissue that integrates mechanical and biological stimuli generated by the hemodynamic load and by circulating molecular factors. It is naturally developed to control vascular homeostasis acting as a selective barrier between the vessel's lumen and the surrounding tissues, preventing thrombosis, modulating the response to infective agents and inflammatory insults, controlling the interaction with circulating immune cells, and promoting vascular repair. ${ }^{2,3}$

The physiological function of endothelial tissues requires integrity of the cell monolayer, which is ensured by the cytoskeleton, the junctional complexes, and the adhesions to the extracellular matrix: i.e., the focal adhesions (FA). In mature endothelia, individual ECs are laterally connected to their neighbors by cell to cell junctions. ${ }^{4}$ At the basal side of ECs, integrin-based FAs establish the connection between the substrate and the contractile cell machinery, through the function of paxillin and other proteins. ${ }^{5}$ Beyond being simple sites of anchoring, these connections can transmit force, relay intracellular signals, and act in concert to support the adaptive response to biochemical and physical stimuli. ${ }^{1,2,6}$

Cellular aging is associated with morphological and functional alterations of individual cells as well as multicellular ensembles and represents a major risk factor for several chronic conditions. ${ }^{7,8}$ This transformation is particularly evident in the human vasculature, ${ }^{9}$ where the endothelial function decays progressively with age, in a process of tissue senescence that typically accompanies cardiovascular disease. ${ }^{10}$ At the cellular level, endothelial senescence results from a sustained accumulation of oxidative and inflammatory stress, leading to genetic and epigenetic alterations. ${ }^{11}$ Specifically, senescent ECs display an altered morphology, phenotypic changes, DNA damage, arrest of the cell cycle, and deregulation of metabolic pathways. ${ }^{12}$ The cell body is typically enlarged and flattened. ${ }^{9,13}$ In addition, inflammatory signaling via nuclear factor $\mathrm{kB}(\mathrm{NF}-\mathrm{kB})^{14,15}$ is activated in response to circulating molecules such as TNF- $\alpha$, typically detected in cardiovascular patients ${ }^{16}$ and in animal models of senescence. ${ }^{17}$ When they are present in sufficient number within a monolayer, senescent ECs can compromise the mechanical and functional stability of the entire tissue.

Although they have been less explored, mechanical and physical signals are deeply involved in regulating the delicate equilibrium between endothelial function and dysfunction. Locally, these factors overlap with the proinflammatory milieu generated by circulating molecules. Specifically, flow-generated wall shear stress (WSS) is a master determinant of endothelial phenotype. ${ }^{18}$ The flow intensity and directionality regulate cell polarization and functional tissue reorganization. ${ }^{19-23}$ The WSS magnitude (venous or arterial) directly influences the cell to cell junction dynamics, modulating tissue permeability in response to inflammatory insults. ${ }^{24}$ As a result, disturbed

Received: January 6, 2021

Revised: $\quad$ May 10, 2021

Published: June 3, 2021

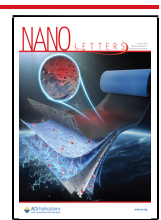


a

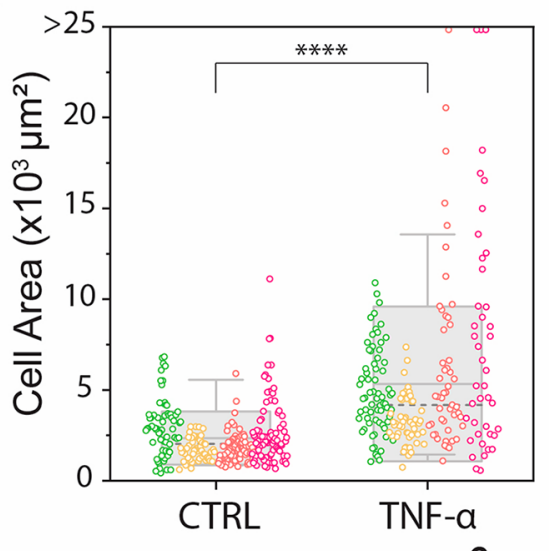

b

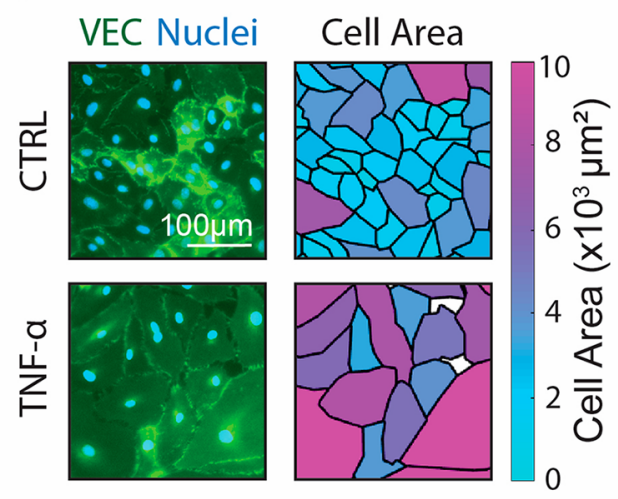

C

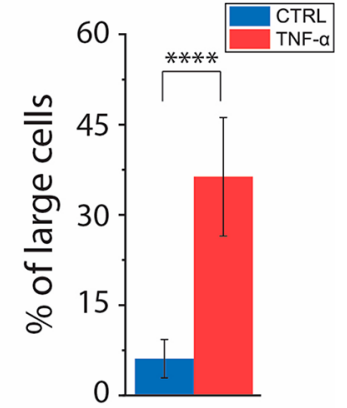

d

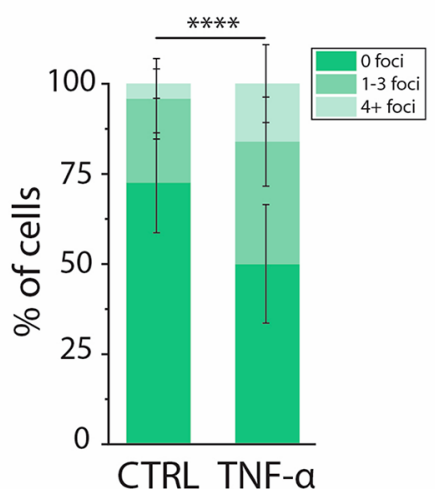

e Nuclei
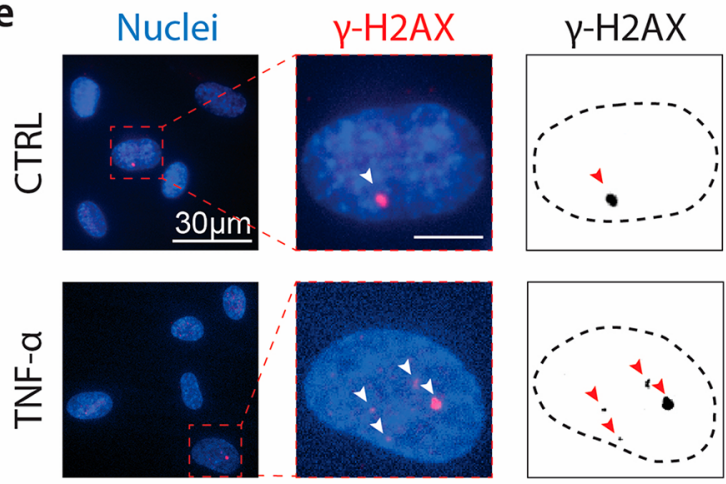

f

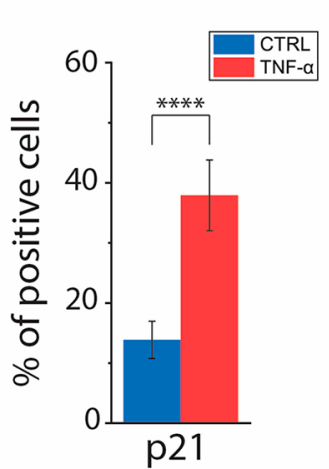

g Nuclei
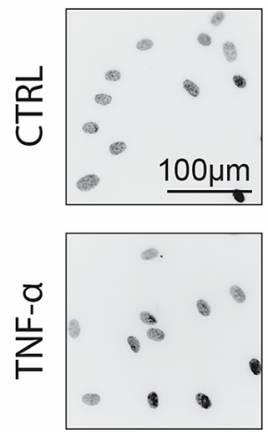

p21
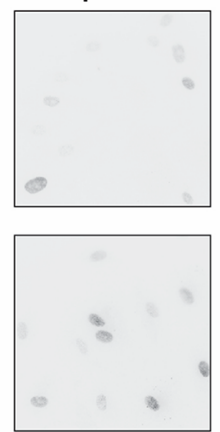

j

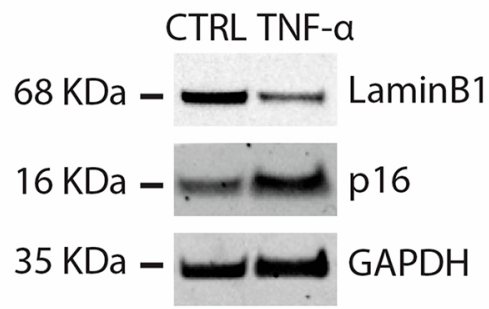

h

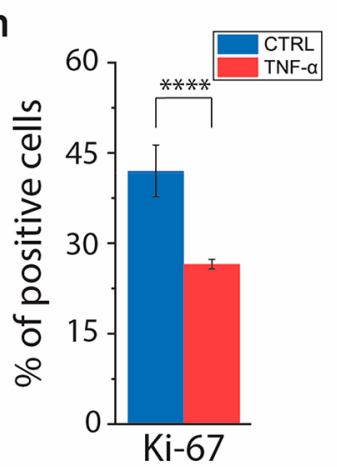

i Nuclei

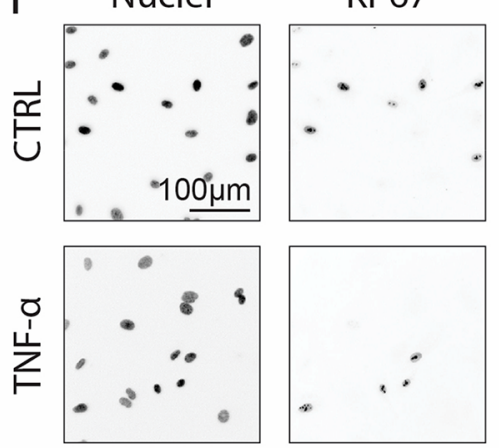

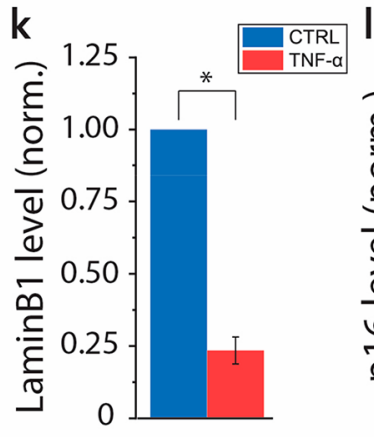

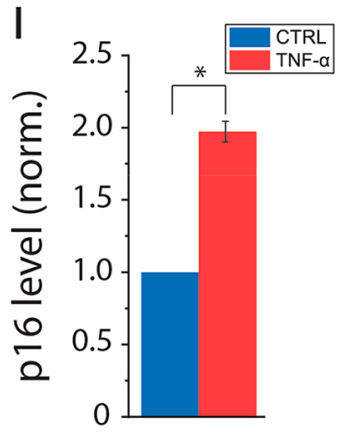

Figure 1. An in vitro model of inflammatory endothelial senescence. $(\mathrm{a}-\mathrm{c})$ Morphological changes. (a) The average cell area is increased upon treatment with TNF- $\alpha\left(n>210\right.$ per condition, $\left.n^{\prime}=4\right)$. Data from independent experiments are displayed as empty circles. (b) Representative immunofluorescence images of vascular endothelial cadherin (VEC, green) and nuclei (DAPI, blue) of control ECs (CTRL, upper panel) and ECs treated with TNF- $\alpha$ (TNF- $\alpha$, lower panel), cell profiles with color-coded cell area, as encoded by the color scale bar. (c) Percentage of large cells $\left(>5000 \mu \mathrm{m}^{2}\right)$ in the control and treated populations $\left(n>210\right.$ per condition, $\left.n^{\prime}=4\right)$. (d, e) DNA damage. (d) Percentage of cells presenting multiple $\gamma$-H2AX foci in the control and treated population $\left(n>165\right.$ per condition, $\left.n^{\prime}=3\right)$. (e) Representative immunofluorescence images of nuclei (DAPI, blue) and foci of DNA damage $(\gamma$-H2AX, red) in control (CTRL, upper panel) and cells treated with TNF- $\alpha$ (TNF- $\alpha$, lower panel). Corresponding magnified view of representative cell nuclei with overlapped signals (middle) and inverted grayscale signal of DNA damage with cell 
Figure 1. continued

profile highlighted by a dashed line (right). The scale bar is $10 \mu \mathrm{m}$. Arrowheads point to individual $\gamma$-H2AX foci. (f -1 ). Altered cell cycle progression. (f) Percentage of $\mathrm{p} 21$ positive cells in control and the treated (day 5) population $\left(n>305\right.$ per condition, $\left.n^{\prime}=4\right)$. (g) Representative immunofluorescence of the cell nuclei (inverted greyscale signal) and p21 positive cells (inverted greyscale signal) of control ECs (CTRL, upper panel) and ECs treated with TNF- $\alpha$ (TNF- $\alpha$, lower panel). (h) Percentage of Ki-67 positive cells in control and treated (day 5) population ( $n>$ 1970 per condition, $n^{\prime}=3$ ). (i) Immunofluorescence images of the cell nuclei (inverted greyscale image) and $K_{\mathrm{i}}-67$ positive cells (inverted greyscale image) of control ECs (CTRL, upper panel) and ECs treated with TNF- $\alpha$ (TNF- $\alpha$, lower panel). (j) Western blot analysis of LaminB1 and p16 expression and $(\mathrm{k}, \mathrm{l})$ corresponding quantifications $\left(n^{\prime}=4\right.$ for LaminB1, $n^{\prime}=3$ for p16). The expression of the housekeeping gene GAPDH was used as a reference. All values in bar plots are reported as mean \pm SEM, the population means are reported as a horizontal line inside the boxplot, the population medians are reported as a dark gray horizontal dashed line inside the boxplot, the vertical length of the box represents the double of the SD and whiskers report the 5th-95th percentile. $*_{p}<0.05, * * * * p<0.0001$.

hemodynamic conditions are among the signals supporting aging in ECs. ${ }^{10,25}$

To address the biophysical differences between young and aged ECs, we applied a protocol ${ }^{26}$ to generate an in vitro model of endothelial senescence. Specifically, low-passage HUVECs were exposed to the proinflammatory molecule TNF- $\alpha$ (Figure S1a). The effect of TNF- $\alpha$ was evaluated through the morphological variations induced in the treated population. ${ }^{9,13}$ After 5 days of treatment the cell area was, on average, 2.3 times larger than that in the untreated control $\left(2354 \pm 87 \mu \mathrm{m}^{2}\right.$ for control and $5335 \pm 294 \mu \mathrm{m}^{2}$ for treated ECs with peaks above $10000 \mu \mathrm{m}^{2}$; Figure $1 \mathrm{a}-\mathrm{c}$ ).

In addition, the biochemical hallmarks of endothelial aging were analyzed. These included the accumulation of DNA damage, the cell cycle arrest, and the proinflammatory signaling. ${ }^{27}$ Immunostaining for $\gamma$-H2AX was used to mark foci of DNA double-strand breaks. ${ }^{28}$ The fraction of cells with multiple $(\geq 3)$ foci of DNA damage was increased 4-fold in the treated population $(16 \pm 11 \%$ vs $4 \pm 4 \%$ in the control; Figure $1 \mathrm{~d}, \mathrm{e})$.

Cell cycle progression was evaluated using different molecular reporters. First, the expression of cyclin-dependent kinase inhibitors, $\mathrm{p} 21^{\mathrm{CIP} 1}(\mathrm{p} 21)$ and $\mathrm{p} 16^{\mathrm{INK} 4 \mathrm{~A}}(\mathrm{p} 16)$, activated by the $\mathrm{p} 21 / \mathrm{p} 53$ and $\mathrm{p} 16 / \mathrm{Rb}$ pathway in response to DNA damage was measured. ${ }^{12}$ Treated cells exhibited increased levels of p21 ( $38 \pm 6 \%$ vs $14 \pm 3 \%$ in the control; Figure $1 \mathrm{f}, \mathrm{g}$ ), as well as a 2 -fold higher expression of p16 (Figure $1 \mathrm{j}, 1$ ). In addition, the cell cycle progression of subconfluent ECs, assessed by $\mathrm{Ki}-67$ expression, confirmed a reduction of proliferation in response to TNF- $\alpha$ ( $42 \pm 4 \%$ for control and $27 \pm 1 \%$ for treated cells; Figure 1h,i). Finally, senescent cells displayed reduced expression of the nuclear envelope protein LaminB1 (4-fold reduction; Figure 1j,k), which regulates heterochromatin organization. ${ }^{29}$

The inflammatory response was monitored by assessing NF$\mathrm{kB}$ activation. ${ }^{30,31} \mathrm{~A}$ peak in the nuclear localization of NF-kB was detected early upon TNF- $\alpha$ administration (35\% higher than control) and was sustained for the entire duration of the treatment (Figure S1g,h).

To confirm the stability of the induced phenotype, senescence markers were reassessed after 3 days of TNF- $\alpha$ treatment discontinuation. While the activation of proinflammatory signaling rapidly tapered (Figure S1g), both the morphological alterations and increased levels of DNA damage remained unaltered, with the senescent population featuring larger cells ( 3 times larger than in the control; Figure $S 1 b-d$ ) and extensive DNA damage (Figure S1e,f). Importantly, the extent of DNA damage did not correlate with the cell size. This result is well in line with recent reports indicating that genetic alterations are at the roots of the senescent $\mathrm{drift}^{32}$ and thus constitute a prerequisite for the ensuing morphological and functional changes.

Previous studies reported the morphological changes and biochemical profile of senescent ECs featuring shortened telomeres, enlargement of the cell body, extended DNA damage, increased mRNA levels of p16 and p21, reduction in proliferation, and decreased levels of LaminB1 protein expression. ${ }^{33-38}$

To further validate the adopted protocol, ${ }^{26}$ we compared the effects of proinflammatory stimulation to those of replicative aging. ${ }^{33,35,36}$ Replicative senescence was obtained by expanding HUVECs for 17 population doublings, corresponding to 1319 passages in vitro, and monitoring their proliferative potential reduction. The resulting population had significantly shortened telomeres (Figure S2). On the other hand, senescent cells obtained by treatment with TNF- $\alpha$ (Figure S1a) featured fulllength telomeres (Figure S2). Importantly, the two senescent populations displayed similar morphological changes and DNA damage. Replicative senescence involved a significant increase in the cell area, with a significant number of cells being larger than $5000 \mu \mathrm{m}^{2}$ (Figure S3a,b). DNA damage was also increased, with $75 \%$ of cells having DNA double-strand breaks, as highlighted by multiple foci of $\gamma$-H2AX accumulation (Figure S3c,d). Altogether these data indicate that, despite the genotypic differences between cells obtained by proinflammatory stimulation or prolonged replication, common features are present in the two populations. Size increase and extensive genetic damage along with the markers of senescence appear even with preserved telomeres.

Endothelial monolayers sense and respond to flow by collectively adapting individual cell shape, orientation, and polarization. When they are exposed to physiological hemodynamic conditions, ${ }^{39}$ ECs elongate and align their body along the direction of flow. ${ }^{40-42}$ At the same time, cell polarization is rotated in the counterflow direction, with the coordinated translocation of the Golgi apparatus upstream of the cell nucleus. ${ }^{43}$

We exploited a custom-developed parallel plate flow chamber $^{40}$ to evaluate the adaptation to flow of monolayers generated by senescent ECs. In these experimental settings, mature endothelia grown under static conditions were exposed to a steady-state, fully developed laminar flow, yielding a WSS of $1.4 \mathrm{~Pa}$ for $16 \mathrm{~h}$ (Videos S1 and S2). The collective response was evaluated by measuring the end point cell density, elongation, orientation, and polarization and comparing them with control values obtained in mature endothelia formed by young and healthy ECs (Figure 2). Individual cell polarization was defined as the angle between the vector starting from the cell nucleus and ending at the Golgi apparatus and the direction of flow (Figure 2f). 

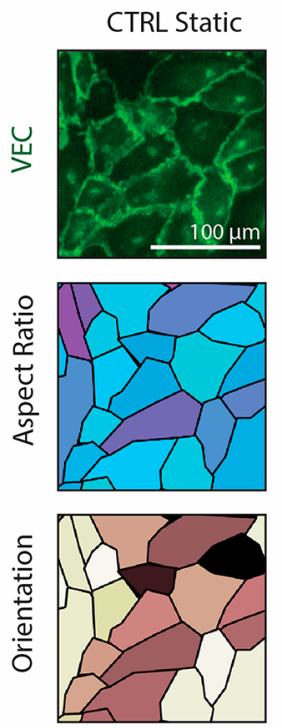

d
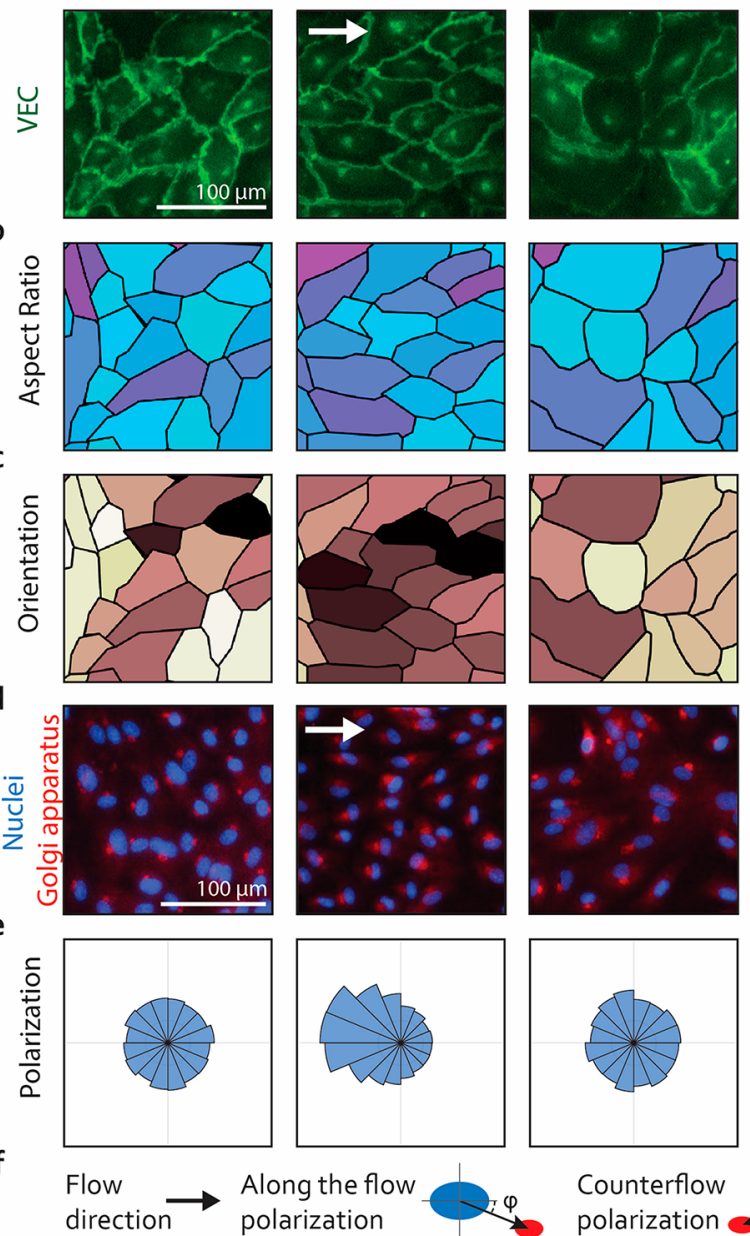

Counterflow

polarization
SEN 1.4 Pa
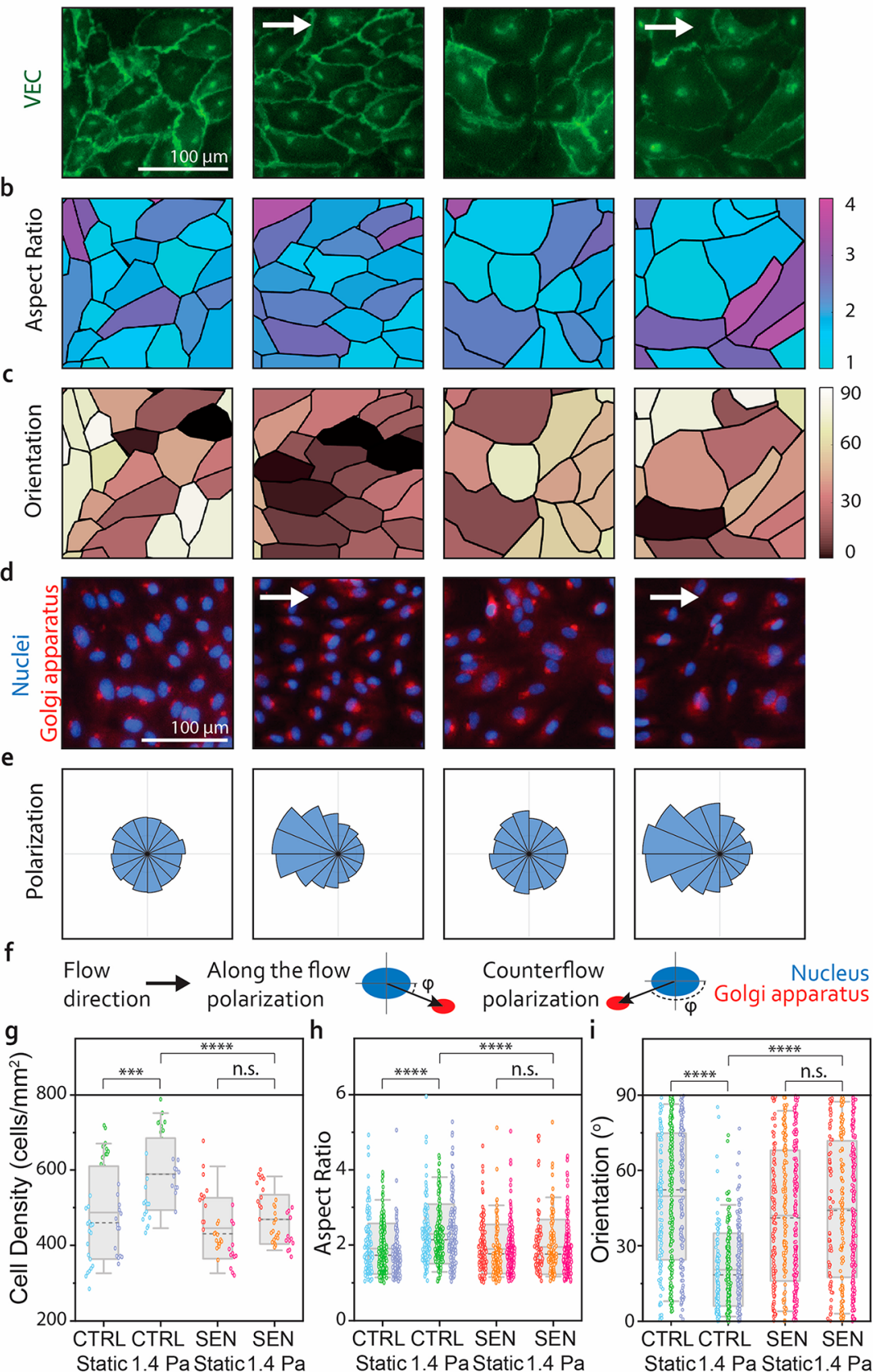

Figure 2. Response of EC monolayer to physiological flow. (a) Analysis of EC monolayer. Immunofluorescent analysis of control (CTRL) and senescent (SEN) cell monolayers in the absence of flow (Static) and upon exposure to flow, leading to a WSS value of $1.4 \mathrm{~Pa}(1.4 \mathrm{~Pa}$ ). The analysis was performed after $16 \mathrm{~h}$ of exposure to flow reporting immunostaining of vascular endothelial cadherin (VEC, green). The white arrow indicates the flow direction. (b) Cell shape adaptation to flow. Visual map of cell aspect ratio (as encoded by the color scale bar). (c) Cell alignment to flow. Visual map of cell orientation (as encoded by the color scale bar). (d) Cell polarization in response to flow. Immunofluorescent analysis of control (CTRL) and senescent (SEN) monolayers in the absence of flow (Static) and upon exposure to flow, leading to a WSS value of $1.4 \mathrm{~Pa}$ for $16 \mathrm{~h}$ (1.4 $\mathrm{Pa}$ ). Immunostaining of nuclei (blue) and Golgi apparatus (red). The white arrow indicates the flow direction. (e) Quantification of cell polarization reported as the radial distribution of polarization angle $\left(n>6200\right.$ per condition, $\left.n^{\prime}=3\right)$. (f) Working definition of polarization angle. Quantitative analysis of $(\mathrm{g})$ cell density $\left(n_{\text {field of view }}>35\right.$ per condition, $\left.n^{\prime}=3\right)$, (h) aspect ratio $\left(n>390\right.$ per condition, $\left.n^{\prime}=3\right)$, and (i) orientation $\left(n>390\right.$ per condition, $\left.n^{\prime}=3\right)$ in control (CTRL) and senescent (SEN) monolayers in the absence of flow (Static) and upon exposure to flow, leading to a WSS value of $1.4 \mathrm{~Pa}$ for $16 \mathrm{~h}(1.4 \mathrm{~Pa})$. Data from independent experiments are displayed as empty circles. In box plots the population means are reported as a line inside the boxplot, the population medians are reported as a dark gray horizontal dash line inside the boxplot, the vertical length of the box represents the double of the SD, and whiskers report the 5th-95th percentile. $* * * p<0.001, * * * * p<0.0001$.

ECs in control endothelia monolayer exhibited an increased aspect ratio $(2.3 \pm 0.78$ under flow and $1.9 \pm 0.67$ under static conditions; Figure 2a,b,h) and the collective reorientation of the cell body along the direction of flow (from $49 \pm 25^{\circ}$ to 20 $\pm 14^{\circ}$; Figure $\left.2 \mathrm{a}, \mathrm{c}, \mathrm{i}\right)$, while preserving a high cell density (487 \pm 123 cells $/ \mathrm{mm}^{2}$ before and $590 \pm 96$ cells $/ \mathrm{mm}^{2}$ after the flow; Figure $2 \mathrm{~g}$ ). In contrast, exposure to flow did not alter the cell morphology or the organization of endothelia formed by 
a

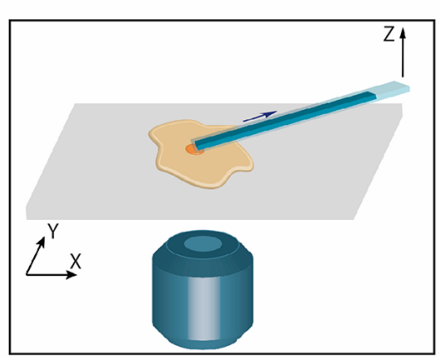

d

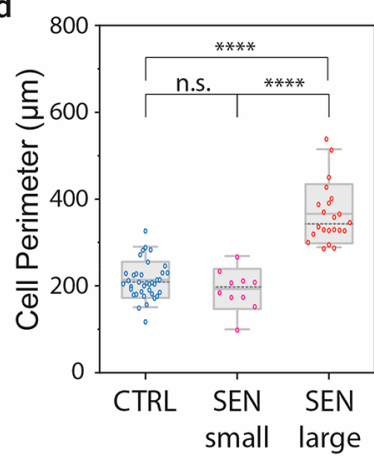

b

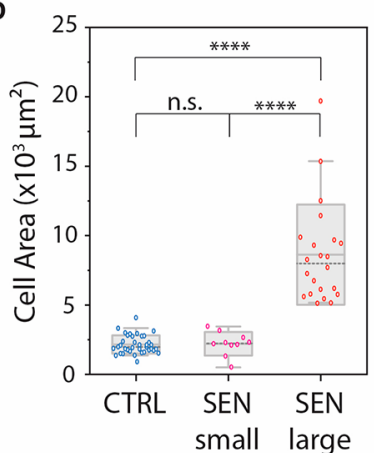

e

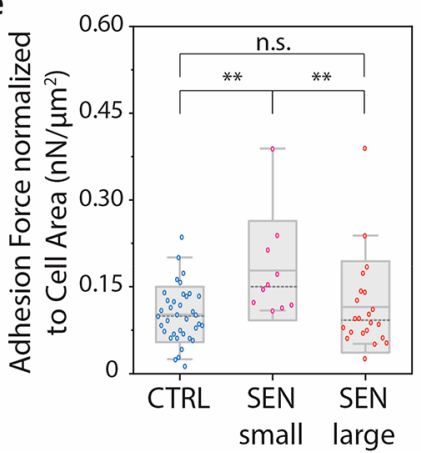

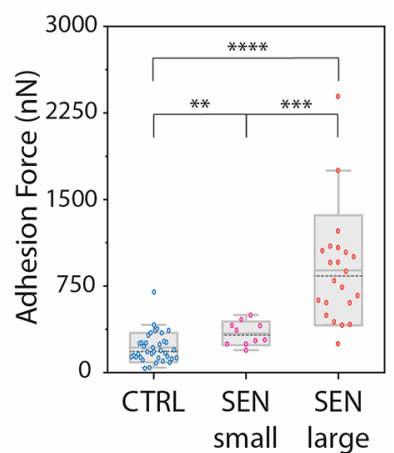

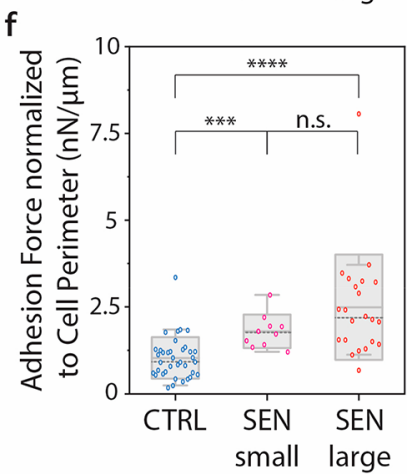

Figure 3. Maximum cell adhesion force of individual ECs. (a) Schematic representation of FluidFM experiments. (b) Quantification of adhesion force in control (CTRL) and senescent small (SEN small, $<5000 \mu \mathrm{m}^{2}$ ) and large (SEN large, $\geq 5000 \mu \mathrm{m}^{2}$ ) cells. Corresponding quantification of (c) the cell area and (d) perimeter. Quantification of adhesion force normalized to (e) the cell area and (f) the cell perimeter. $\left(n_{\mathrm{CTRL}}=37\right.$, $n_{\mathrm{SEN}}=$ $\left.32, n_{\mathrm{SEN}, \text { small }}=10, n_{\mathrm{SEN}, \text { large }}=22, n^{\prime}=6\right)$. Data from independent experiments are displayed as empty circles. In boxplots the population means are reported as a horizontal line inside the boxplot, the population medians are reported as a dark gray horizontal dashed line inside the boxplot, the vertical length of the box represents the double of the SD, and whiskers report the 5th-95th percentile. $* * p<0.01, * * * p<0.001, * * * * p<0.0001$.

senescent cells. Here, ECs maintained a round shape $(2.0 \pm$ 0.72 under flow and $1.9 \pm 0.65$ under static conditions; Figure $2 \mathrm{a}, \mathrm{b}, \mathrm{h})$ and random orientation $\left(42 \pm 26^{\circ}\right.$ before and $45 \pm$ $27^{\circ}$ after flow; Figure 2a,c,i). The cell density was similarly not perturbed by the flow $\left(446 \pm 80\right.$ cells $/ \mathrm{mm}^{2}$ before and $467 \pm$ 65 cells $/ \mathrm{mm}^{2}$ after the flow; Figure $2 \mathrm{~g}$ ). This is a clear departure from the control cell behavior, demonstrating that senescent endothelia lose their ability to actively remodel and adapt their shape in response to flow. On the other hand, a clear counterflow cell polarization was achieved under both conditions (Figure 2d-f). This adaptive response is therefore independent of the concomitant cell shape remodeling and reorientation and is surprisingly not compromised by aging.

Adaptation of endothelial monolayers to flow requires the generation of cellular tractions and their transmission to the substrate. $^{44}$ The compromised response of senescent ECs (Figure 2) points to an alteration of this process. Their mechanical interaction with the substrate was therefore quantitatively evaluated. The maximum normal adhesion force, i.e., the resistance of individual cells to a vertically applied pull, is efficiently measured by FluidFM-based singlecell force spectroscopy (SCFS; Figure 3a). ${ }^{45-47}$

FluidFM measurements (Figure 3) were performed on isolated ECs. ${ }^{45}$ In fact, the interaction with neighboring cells, as in a monolayer, increases the measurement complexity and does not allow the direct comparison of adhesion force in different populations. ECs were allowed to fully adhere and spread under unconfined conditions. In these experimental settings, they featured an average size larger than that in confluent monolayers. ${ }^{48}$ This variation is displayed in the Table S1.
Specifically, the tested senescent cells were subdivided into two groups, including small $\left(<5000 \mu \mathrm{m}^{2}\right.$; average area of 2207 $\pm 267 \mu \mathrm{m}^{2}$; Figure $3 \mathrm{~b}$ and Table S1) and large cells $(\geq 5000$ $\mu \mathrm{m}^{2}$; average area $8626 \pm 769 \mu \mathrm{m}^{2}$; Figure $3 \mathrm{~b}$ and Table S1). Small senescent ECs featured an average area and circularity $(0.73 \pm 0.05$; Figure S4) similar to those of the control population $\left(2155 \pm 108 \mu \mathrm{m}^{2}\right.$ and $0.61 \pm 0.03$, respectively; Figure 3b, Figure S4, and Table S1). Cells in the large senescent group reached a size of $5000 \mu \mathrm{m}^{2}$ and beyond and featured higher circularity $(0.78 \pm 0.02$; Figure $3 \mathrm{~b}$ and Figure S4).

The values of cell size for the control and senescent populations (Figure 1a and Figure S1b) are distributed with high skewness and do not fulfill the requirements of a normal ordering. The asymmetric range is instead well captured by a log-normal distribution ${ }^{49}$ ( $p$ value $>0.05$; Figure S5). Cells included in the FluidFM experiments (Figure 3) were selected to represent the entire distribution range, as displayed in the respective histograms included in Figure S5. Therefore, the resulting standard deviations reflect the population distribution, yielding a large variability around the mean.

All senescent ECs had significantly elevated adhesion forces $(341 \pm 32,887 \pm 102$, and $217 \pm 21 \mathrm{nN}$ in small senescent, large senescent, and control ECs, respectively; Figure 3c). In smaller senescent ECs, the stronger connection to the substrate was naturally translated into an increment of adhesion force normalized to cell area and perimeter (Figure 3e,f).

In large senescent ECs, however, the measured adhesion force values normalized to cell area were equal to the control, as the increments in size and adhesion compensated each other (Figure 3e). The concomitant increase in circularity (Figure 
a

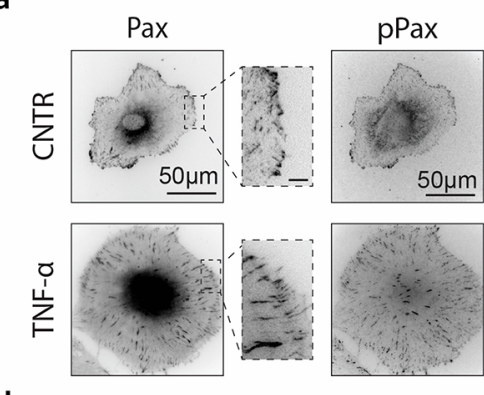

d
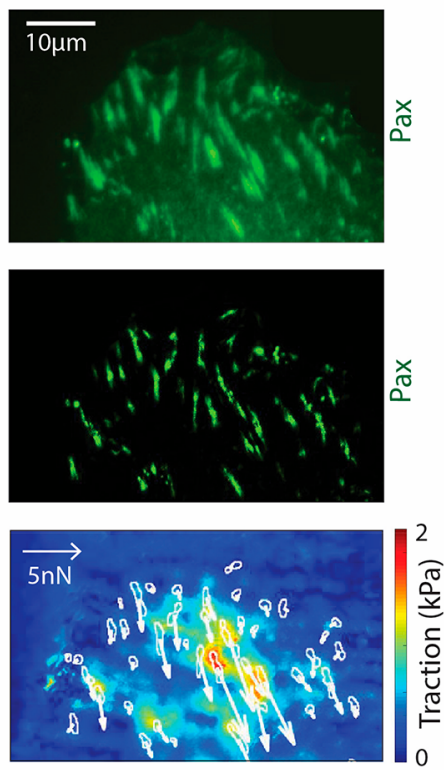

b

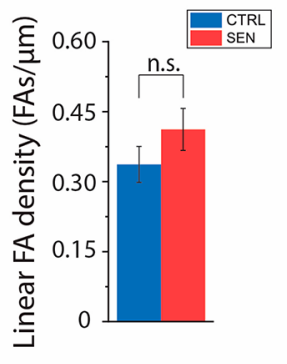

e

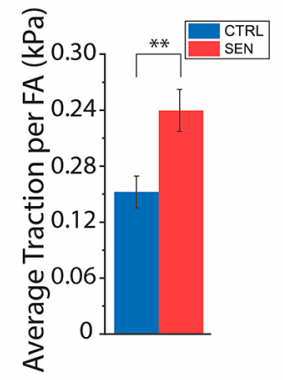

g

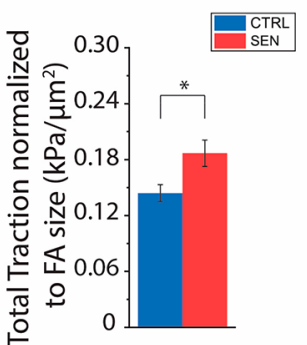

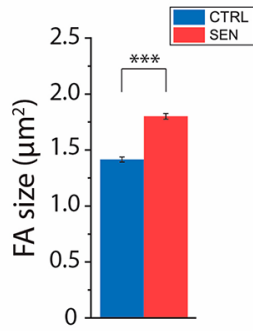

f

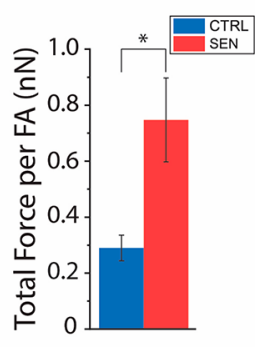

h

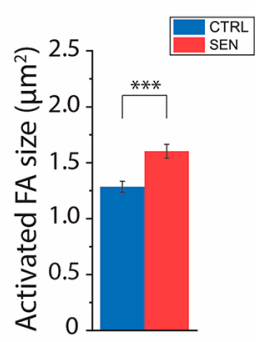

Figure 4. Traction forces and focal adhesion maturation. (a) Individual focal adhesions revealed by immunostaining for paxillin (Pax, inverted greyscale signal) in control (CTRL) and senescent (SEN) cells and magnification of the cell periphery (middle) The scale bar is $5 \mu$ m. Corresponding immunostaining of phosphorylated paxillin (pPax, right). (b) Density of FA established by control (CTRL) and senescent (SEN) cells. The number of FAs is normalized to the cell perimeter $(n>1200$ per condition). (c) Quantification of FA area, based on the expression of paxillin, in control (CTRL) and senescent (SEN) cells $(n>380$ per condition). (d) Combined confocal traction force microscopy (cTFM) analysis of FA density, size, and tractions. Representative immunofluorescence image of paxillin (Pax, green, upper panel) and analysis of the paxillin signal to get the FA profile (Pax, green, middle panel). Corresponding map of tractions with overlay of FAs profile (white outlines, FA profile, lower panel). (e) Total traction per FA in control (CTRL) and senescent (SEN) cells ( $n>700$ per condition). (f) Quantification of total force per FA in control (CTRL) and senescent (SEN) cells $(n>700$ per condition). (g) Quantification of total traction per FA normalized to the corresponding FA area ( $n>700$ per condition). (h) Quantification of activated FA size, based on the expression of phosphorylated paxillin (pPax), in control (CTRL) and senescent (SEN) cells $\left(n>380\right.$ per condition). Results from a representative experiment are presented $\left(n^{\prime}=3\right)$, and all values are reported as mean \pm SEM. $*_{p}<0.05, * * p<0.01, * * * p<0.001$.

S4) was reflected in a dramatic increase of the force over the cell perimeter (Figure $3 \mathrm{f}$ ).

The increased adhesion force measured in senescent ECs (Figure 3c) could be the result of a higher number of FAs established with the substrate and/or of the increased mechanical maturation of individual adhesion points. ${ }^{50}$

To evaluate these hypotheses, we analyzed the biochemical and mechanical properties of FAs in young and senescent ECs. Specifically, the FA density and their size were measured on the basis of the distribution of paxillin, a major cytoplasmic component of adhesion plaques (Figure $4 a) .{ }^{51}$ While the linear FA densities at the cell periphery were similar under the two conditions $(0.3 \pm 0.04 \mathrm{FAs} / \mu \mathrm{m}$ for control and $0.4 \pm 0.05$ FAs $/ \mu \mathrm{m}$ for senescent cells; Figure $4 \mathrm{~b})$ the area of individual FAs was significantly higher in senescent ECs $\left(1.4 \pm 0.1 \mu \mathrm{m}^{2}\right.$ for control and $1.8 \pm 0.1 \mu \mathrm{m}^{2}$ for senescent cells; Figure $4 \mathrm{c}$ ).

To assess whether the larger size of FAs was linked to an increased mechanical maturity, we measured the force transmitted at the level of individual adhesion points by means of confocal traction force microscopy (cTFM), quantifying tractions and correlating them with the respective FA. $^{52-55}$

On average, individual FAs established by senescent cells exerted 1.6-fold higher average tractions $(0.15 \pm 0.02 \mathrm{kPa}$ for control and $0.24 \pm 0.02 \mathrm{kPa}$ for senescent cells; Figure $4 \mathrm{e}$ ) and 2.6-fold higher force on the substrate $(0.29 \pm 0.05 \mathrm{nN}$ for control and $0.75 \pm 0.15 \mathrm{nN}$ for senescent cells; Figure 4f).

The total force per FA area depends on the mechanical maturation of adhesion sites ${ }^{50}$ and was similarly enhanced in senescent ECs $\left(0.14 \pm 0.01 \mathrm{kPa} / \mu \mathrm{m}^{2}\right.$ for control and $0.19 \pm$ $0.01 \mathrm{kPa} / \mu \mathrm{m}^{2}$ for senescent cells; Figure $4 \mathrm{~g}$ ). Consistently, phosphorylated paxillin ( $\mathrm{pPax})$, a biochemical marker of the FA mechanical activity, ${ }^{51}$ was enriched in the FAs formed by senescent cells $\left(1.3 \pm 0.1 \mu \mathrm{m}^{2}\right.$ for control and $1.6 \pm 0.1 \mu \mathrm{m}^{2}$ for senescent cells; Figure 4h). 
Biological senescence in ECs is typically described as the deviation from a young and healthy phenotype, which results from the accumulation of metabolic, genetic, and epigenetic damages. ${ }^{12}$ Noxious signals of a different nature are responsible for and sustain this drift. Inflammatory circulating molecules have a systemic effect, while a disturbance of the local hemodynamic load creates regions in which groups of cells age rapidly and expose the vasculature to atherosclerosis. ${ }^{25,56}$ The mechanism by which individual cell senescence compromises the overall function of endothelial tissues likely entails the disruption of collective processes allowing mature monolayers to adapt to flow. ${ }^{11,12}$ These include the coordinated remodeling of cell shape and polarization, which is consistently observed along the vascular tree. ${ }^{25,43}$

The first element to consider is the altered morphology of senescent ECs that can be observed both in vivo ${ }^{11,57}$ and is reproduced in vitro (Figure $1 \mathrm{a}-\mathrm{c}$ and Figures S1b-d and $3 \mathrm{a}, \mathrm{b}$ ). Senescence typically promotes a multifold size increase (Figure $1 \mathrm{a}, \mathrm{b}$ and Figures S1b,c and 3a). In the context of a confluent monolayer, each senescent cell will engage the space previously occupied by several (e.g., two to five) healthy cells (Figure $1 \mathrm{a}, \mathrm{b}$, Figures $\mathrm{S} 1 \mathrm{~b}, \mathrm{c}$ and $3 \mathrm{a}$ ). In this way, senescence decreases the local cell density and the sites where cell to cell junctions can be established, reducing the possibility of their dynamic remodeling.

The active rearrangement of junctional sites and adhesions to the substrate is needed during endothelial repair and adaptation to the local hemodynamic conditions. ${ }^{24,40,44}$ In particular, the orientation of cell ensembles requires a coordinated remodeling of cell to cell and cell to substrate adhesions, to preserve the monolayer integrity while collectively acquiring an anisotropic configuration. ${ }^{58,59}$

When they are exposed to flow, senescent monolayers are unable to adapt and remain isotropic (Figure 2a,b,h), suggesting that either the flow sensing mechanism or the ensuing response is impaired. Interestingly, senescent ECs can polarize in response to the flow directionality (Figure 2d,e), a process requiring the translocation of the Golgi apparatus upstream of the nucleus and supporting the cell migration in the counter flow direction. ${ }^{43}$ This observation suggests that flow sensing is indeed retained; yet the processes of coordinated reorientation cannot be fulfilled.

To attain a collective change in shape and orientation, cells must coordinate force generation and transmission to the substrate with the transient and partial disassembly of the connections established at the basal side: i.e., the FAs. ${ }^{5,6,44}$ Senescent ECs feature an increased mechanical maturity of adhesion points, which transmit larger tractions and forces to the substrate (Figure $4 \mathrm{~d}-\mathrm{h}) .^{60}$ These cells generate and apply more contractility. When these cells are probed for their maximum resistance to normal tractions, they also demonstrate a stronger adhesion to the substrate (Figure 3c), which is directly proportional to their increased perimeter (Figure 3d,f). Stronger adhesion to the substrate may impede the dynamic processes required for collective cell orientation and alignment to flow.

In vivo, endothelial senescence appears under nonphysiological hemodynamic conditions, which are typically found at vessel bifurcations, in correspondence with arterial stenosis, or downstream of cardiovascular implants. ${ }^{10,25,57,61,62}$ Here, the combination of proinflammatory signaling and disturbed flow endangers endothelial function and integrity, promoting the migration of ECs away from the noxious stimulus ${ }^{63,64}$ and/or their progressive deadhesion and death. ${ }^{65}$ Endothelial denudation (i.e., the loss of coverage by a confluent endothelium leading to the exposure of the underlying substrate) and the ensuing chronic inflammatory process generate hotspots for the formation and maturation of atherosclerotic plaques. ${ }^{10-12,56}$

While the alteration of cell to cell junction distribution and FA dynamics compromises their collective remodeling, senescent ECs may represent an extreme measure to avoid local tissue denudation. Larger individual cells may serve to fill the space generated by the progressive cellular loss. The establishment of stronger connections may increase their resistance to detrimental hemodynamic conditions. In this way, stronger adhesions to the substrate (Figure 3) and increased contractility (Figure 4) may allow the endothelium to delay denudation by locally enforcing a static and resistant coverage.

\section{ASSOCIATED CONTENT}

\section{(5) Supporting Information}

Video S1. Video S2. The Supporting Information is available free of charge at https://pubs.acs.org/doi/10.1021/acs.nanolett.1c00064.

Detailed information about materials and methods, accelerated in vitro aging of ECs, telomere shortening, replicative senescence of ECs, circularity of ECs in FluidFM experiments, and cell area distribution (PDF)

Flow experiment, WSS $1.4 \mathrm{~Pa}$, control endothelial monolayer (AVI)

Flow experiment, WSS 1.4 Pa, senescent endothelial monolayer(AVI)

\section{AUTHOR INFORMATION}

\section{Corresponding Authors}

Dimos Poulikakos - Laboratory of Thermodynamics in Emerging Technologies, Department of Mechanical and Process Engineering, ETH Zurich, 8092 Zürich, Switzerland; ○ orcid.org/0000-0001-5733-6478; Email: dpoulikakos@ ethz.ch

Aldo Ferrari - Laboratory of Thermodynamics in Emerging Technologies, Department of Mechanical and Process Engineering, ETH Zurich, 8092 Zürich, Switzerland; Experimental Continuum Mechanics, EMPA, Swiss Federal Laboratories for Material Science and Technologies, 8600 Dübendorf, Switzerland; Institute for Mechanical Systems, Department of Mechanical and Process Engineering, ETH Zurich, 8092 Zürich, Switzerland; 이이.org/0000-00015430-1317; Email: aferrari@ethz.ch

\section{Authors}

Nafsika Chala - Laboratory of Thermodynamics in Emerging Technologies, Department of Mechanical and Process Engineering, ETH Zurich, 8092 Zürich, Switzerland

Silvia Moimas - Laboratory of Thermodynamics in Emerging Technologies, Department of Mechanical and Process Engineering, ETH Zurich, 8092 Zürich, Switzerland

Costanza Giampietro - Experimental Continuum Mechanics, EMPA, Swiss Federal Laboratories for Material Science and Technologies, 8600 Dübendorf, Switzerland; Institute for Mechanical Systems, Department of Mechanical and Process Engineering, ETH Zurich, 8092 Zürich, Switzerland 
Xinyu Zhang - Laboratory of Biosensors and Bioelectronics, Department of Information Technology and Electrical Engineering, ETH Zurich, 8092 Zürich, Switzerland

Tomaso Zambelli - Laboratory of Biosensors and Bioelectronics, Department of Information Technology and Electrical Engineering, ETH Zurich, 8092 Zürich, Switzerland

Vasileios Exarchos - German Heart Center Berlin, Department for Cardiovascular and Thoracic Surgery, 13353 Berlin, Germany

Timo Z. Nazari-Shafti - German Heart Center Berlin, Department for Cardiovascular and Thoracic Surgery, 13353 Berlin, Germany; Berlin Institute of Health at CharitéUniversitätsmedizin Berlin, Center for Regenerative Therapies, 13353 Berlin, Germany

Complete contact information is available at: https://pubs.acs.org/10.1021/acs.nanolett.1c00064

\section{Author Contributions}

N.C. performed all the cell experiments and analyzed the data. S.M. and C.G. supported the design and validation of the cell aging protocols and the flow experiments. X.Z. performed the FluidFM experiments and the corresponding data analysis. T.Z. supervised and supported the FluidFM experiments and data analysis. V.E. and T.Z.N.-S, performed replicative senescence experiments, telomere shortening measurements and the corresponding data analysis. D.P. designed and supervised the study. A.F. designed the study and supervised the cTFM experiments. All authors contributed to the writing of the manuscript.

\section{Notes}

The authors declare no competing financial interest.

\section{ACKNOWLEDGMENTS}

This work is part of the Zurich Heart project under the umbrella of "Hochschulmedizin Zurich" and is supported by the Stavros Niarchos Foundation (SNF). It is also supported by funding from the Swiss National Science Foundation (SNSF) under Grant SNF (205321_188828). X.Z. is supported by EU H2020 Marie Sklodowska-Curie Actions (ITN "SENTINEL” no. 812398). T.Z.N.-S. is a scholar in the BIH Charité Clinician Scientist Program funded by the Charité-Universitätsmedizin Berlin and the Berlin Institute of Health. We thank Gabriele Fontana (ETH, Switzerland) and Alesandro Poli (IFOM, Italy) for their help in Western blot and Maren Husmann, Jonathan Ocampo Chavez, and Simone Tonnicchia (ETH, Switzerland) for their help with the cTFM sample preparation.

\section{REFERENCES}

(1) Lampugnani, M. G.; Dejana, E.; Giampietro, C. Corrigendum: Vascular Endothelial (VE)-Cadherin, Endothelial Adherens Junctions, and Vascular Disease. Cold Spring Harb Perspect Biol. 2017, 9 (10), No. a033720.

(2) Dejana, E. Endothelial cell-cell junctions: happy together. Nat. Rev. Mol. Cell Biol. 2004, 5 (4), 261-70.

(3) Vitorino, P.; Hammer, M.; Kim, J.; Meyer, T. A steering model of endothelial sheet migration recapitulates monolayer integrity and directed collective migration. Mol. Cell. Biol. 2011, 31 (2), 342-50.

(4) Bazzoni, G.; Dejana, E. Endothelial cell-to-cell junctions: molecular organization and role in vascular homeostasis. Physiol. Rev. 2004, 84 (3), 869-901.
(5) Zaidel-Bar, R.; Ballestrem, C.; Kam, Z.; Geiger, B. Early molecular events in the assembly of matrix adhesions at the leading edge of migrating cells. J. Cell Sci. 2003, 116 (22), 4605-4613.

(6) Geiger, B.; Spatz, J. P.; Bershadsky, A. D. Environmental sensing through focal adhesions. Nat. Rev. Mol. Cell Biol. 2009, 10 (1), 21-33.

(7) Kennedy, B. K.; Berger, S. L.; Brunet, A.; Campisi, J.; Cuervo, A. M.; Epel, E. S.; Franceschi, C.; Lithgow, G. J.; Morimoto, R. I.; Pessin, J. E.; Rando, T. A.; Richardson, A.; Schadt, E. E.; Wyss-Coray, T.; Sierra, F. Geroscience: linking aging to chronic disease. Cell 2014, 159 (4), 709-13.

(8) Lopez-Otin, C.; Blasco, M. A.; Partridge, L.; Serrano, M.; Kroemer, G. The hallmarks of aging. Cell 2013, 153 (6), 1194-217.

(9) Tian, X. L.; Li, Y. Endothelial cell senescence and age-related vascular diseases. J. Genet. Genomics 2014, 41 (9), 485-95.

(10) Nazari-Shafti, T. Z.; Cooke, J. P. Telomerase Therapy to Reverse Cardiovascular Senescence. Methodist Debakey Cardiovasc J. 2015, 11 (3), 172-5.

(11) Donato, A. J.; Morgan, R. G.; Walker, A. E.; Lesniewski, L. A. Cellular and molecular biology of aging endothelial cells. J. Mol. Cell Cardiol 2015, 89, 122-135.

(12) Mistriotis, P.; Andreadis, S. T. Vascular aging: Molecular mechanisms and potential treatments for vascular rejuvenation. Ageing Res. Rev. 2017, 37, 94-116.

(13) Unterluggauer, H.; Hutter, E.; Voglauer, R.; Grillari, J.; Voth, M.; Bereiter-Hahn, J.; Jansen-Durr, P.; Jendrach, M. Identification of cultivation-independent markers of human endothelial cell senescence in vitro. Biogerontology 2007, 8 (4), 383-97.

(14) Donato, A. J.; Black, A. D.; Jablonski, K. L.; Gano, L. B.; Seals, D. R. Aging is associated with greater nuclear NF kappa B, reduced I kappa B alpha, and increased expression of proinflammatory cytokines in vascular endothelial cells of healthy humans. Aging Cell 2008, 7 (6), $805-12$.

(15) Taniguchi, K.; Karin, M. NF-kappaB, inflammation, immunity and cancer: coming of age. Nat. Rev. Immunol. 2018, 18 (5), 309324.

(16) Anker, S. D.; Von Haehling, S. Inflammatory mediators in chronic heart failure: An overview. Br. Heart J. 2004, 90 (4), 464470 .

(17) Smith, A. R.; Visioli, F.; Frei, B.; Hagen, T. M. Age-related changes in endothelial nitric oxide synthase phosphorylation and nitric oxide dependent vasodilation: evidence for a novel mechanism involving sphingomyelinase and ceramide-activated phosphatase $2 \mathrm{~A}$. Aging Cell 2006, 5 (5), 391-400.

(18) Campinho, P.; Vilfan, A.; Vermot, J. Blood Flow Forces in Shaping the Vascular System: A Focus on Endothelial Cell Behavior. Front Physiol 2020, 11, 552.

(19) Morgan, J. T.; Pfeiffer, E. R.; Thirkill, T. L.; Kumar, P.; Peng, G.; Fridolfsson, H. N.; Douglas, G. C.; Starr, D. A.; Barakat, A. I. Nesprin-3 regulates endothelial cell morphology, perinuclear cytoskeletal architecture, and flow-induced polarization. Mol. Biol. Cell 2011, 22 (22), 4324-34.

(20) Tzima, E.; Kiosses, W. B.; del Pozo, M. A.; Schwartz, M. A. Localized cdc42 activation, detected using a novel assay, mediates microtubule organizing center positioning in endothelial cells in response to fluid shear stress. J. Biol. Chem. 2003, 278 (33), 31020-3.

(21) Butcher, J. T.; Penrod, A. M.; Garcia, A. J.; Nerem, R. M. Unique morphology and focal adhesion development of valvular endothelial cells in static and fluid flow environments. Arterioscler., Thromb., Vasc. Biol. 2004, 24 (8), 1429-34.

(22) Ostrowski, M. A.; Huang, N. F.; Walker, T. W.; Verwijlen, T.; Poplawski, C.; Khoo, A. S.; Cooke, J. P.; Fuller, G. G.; Dunn, A. R. Microvascular endothelial cells migrate upstream and align against the shear stress field created by impinging flow. Biophys. J. 2014, 106 (2), 366-74.

(23) Michalaki, E.; Surya, V. N.; Fuller, G. G.; Dunn, A. R. Perpendicular alignment of lymphatic endothelial cells in response to spatial gradients in wall shear stress. Commun. Biol. 2020, 3 (1), 57.

(24) Orsenigo, F.; Giampietro, C.; Ferrari, A.; Corada, M.; Galaup, A.; Sigismund, S.; Ristagno, G.; Maddaluno, L.; Koh, G. Y.; Franco, 
D.; Kurtcuoglu, V.; Poulikakos, D.; Baluk, P.; McDonald, D.; Grazia Lampugnani, M.; Dejana, E. Phosphorylation of VE-cadherin is modulated by haemodynamic forces and contributes to the regulation of vascular permeability in vivo. Nat. Commun. 2012, 3, 1208.

(25) Chiu, J. J.; Chien, S. Effects of disturbed flow on vascular endothelium: pathophysiological basis and clinical perspectives. Physiol. Rev. 2011, 91 (1), 327-87.

(26) Khan, S. Y.; Awad, E. M.; Oszwald, A.; Mayr, M.; Yin, X.; Waltenberger, B.; Stuppner, H.; Lipovac, M.; Uhrin, P.; Breuss, J. M. Premature senescence of endothelial cells upon chronic exposure to TNFalpha can be prevented by $\mathrm{N}$-acetyl cysteine and plumericin. Sci. Rep 2017, 7, 39501.

(27) Gorgoulis, V.; Adams, P. D.; Alimonti, A.; Bennett, D. C.; Bischof, O.; Bishop, C.; Campisi, J.; Collado, M.; Evangelou, K.; Ferbeyre, G.; Gil, J.; Hara, E.; Krizhanovsky, V.; Jurk, D.; Maier, A. B.; Narita, M.; Niedernhofer, L.; Passos, J. F.; Robbins, P. D.; Schmitt, C. A.; Sedivy, J.; Vougas, K.; von Zglinicki, T.; Zhou, D.; Serrano, M.; Demaria, M. Cellular Senescence: Defining a Path Forward. Cell 2019, 179 (4), 813-827.

(28) Kuo, L. J.; Yang, L. X. Gamma-H2AX - a novel biomarker for DNA double-strand breaks. In Vivo 2008, 22 (3), 305-309.

(29) Shimi, T.; Butin-Israeli, V.; Adam, S. A.; Hamanaka, R. B.; Goldman, A. E.; Lucas, C. A.; Shumaker, D. K.; Kosak, S. T.; Chandel, N. S.; Goldman, R. D. The role of nuclear lamin B1 in cell proliferation and senescence. Genes Dev. 2011, 25 (24), 2579-93.

(30) Mussbacher, M.; Salzmann, M.; Brostjan, C.; Hoesel, B.; Schoergenhofer, C.; Datler, H.; Hohensinner, P.; Basilio, J.; Petzelbauer, P.; Assinger, A.; Schmid, J. A. Cell Type-Specific Roles of NF-kappaB Linking Inflammation and Thrombosis. Front Immunol 2019, 10, 85.

(31) Stefopoulos, G.; Giampietro, C.; Falk, V.; Poulikakos, D.; Ferrari, A. Facile endothelium protection from TNF-alpha inflammatory insult with surface topography. Biomaterials 2017, 138, 131-141.

(32) Schumacher, B.; Pothof, J.; Vijg, J.; Hoeijmakers, J. H. J. The central role of DNA damage in the ageing process. Nature 2021, 592 (7856), 695-703.

(33) Bochenek, M. L.; Bauer, T.; Gogiraju, R.; Nadir, Y.; Mann, A.; Schonfelder, T.; Hunig, L.; Brenner, B.; Munzel, T.; Wenzel, P.; Konstantinides, S.; Schafer, K. The endothelial tumor suppressor p53 is essential for venous thrombus formation in aged mice. Blood $A d v$. 2018, 2 (11), 1300-1314.

(34) Eman, M. R.; Regan-Klapisz, E.; Pinkse, M. W.; Koop, I. M.; Haverkamp, J.; Heck, A. J.; Verkleij, A. J.; Post, J. A. Protein expression dynamics during replicative senescence of endothelial cells studied by 2-D difference in-gel electrophoresis. Electrophoresis 2006, 27 (8), 1669-82.

(35) Korybalska, K.; Kawka, E.; Kusch, A.; Aregger, F.; Dragun, D.; Jorres, A.; Breborowicz, A.; Witowski, J. Recovery of senescent endothelial cells from injury. J. Gerontol., Ser. A 2013, 68 (3), 250-7.

(36) Krouwer, V. J.; Hekking, L. H.; Langelaar-Makkinje, M.; ReganKlapisz, E.; Post, J. A. Endothelial cell senescence is associated with disrupted cell-cell junctions and increased monolayer permeability. Vasc. Cell 2012, 4 (1), 12.

(37) Li, R.; Mi, X.; Yang, S.; Yang, Y.; Zhang, S.; Hui, R.; Chen, Y.; Zhang, W. Long-term stimulation of angiotensin II induced endothelial senescence and dysfunction. Exp. Gerontol. 2019, 119, 212-220.

(38) Haendeler, J.; Hoffmann, J.; Diehl, J. F.; Vasa, M.; Spyridopoulos, I.; Zeiher, A. M.; Dimmeler, S. Antioxidants inhibit nuclear export of telomerase reverse transcriptase and delay replicative senescence of endothelial cells. Circ. Res. 2004, 94 (6), 768-75.

(39) Kroll, M. H.; Hellums, J. D.; McIntire, L. V.; Schafer, A. I.; Moake, J. L. Platelets and shear stress. Blood 1996, 88 (5), 15251541.

(40) Franco, D.; Milde, F.; Klingauf, M.; Orsenigo, F.; Dejana, E.; Poulikakos, D.; Cecchini, M.; Koumoutsakos, P.; Ferrari, A.; Kurtcuoglu, V. Accelerated endothelial wound healing on microstructured substrates under flow. Biomaterials 2013, 34 (5), 1488-97.
(41) Levesque, M. J.; Nerem, R. M. The elongation and orientation of cultured endothelial cells in response to shear stress. J. Biomech. Eng. 1985, 107 (4), 341-7.

(42) Malek, A. M.; Izumo, S. Mechanism of endothelial cell shape change and cytoskeletal remodeling in response to fluid shear stress. J. Cell Sci. 1996, 109 (4), 713-726.

(43) Tkachenko, E.; Gutierrez, E.; Saikin, S. K.; Fogelstrand, P.; Kim, C.; Groisman, A.; Ginsberg, M. H. The nucleus of endothelial cell as a sensor of blood flow direction. Biol. Open 2013, 2 (10), 1007-12.

(44) Ting, L. H.; Jahn, J. R.; Jung, J. I.; Shuman, B. R.; Feghhi, S.; Han, S. J.; Rodriguez, M. L.; Sniadecki, N. J. Flow mechanotransduction regulates traction forces, intercellular forces, and adherens junctions. Am. J. Physiol Heart Circ Physiol 2012, 302 (11), H2220-9.

(45) Potthoff, E.; Franco, D.; D’Alessandro, V.; Starck, C.; Falk, V.; Zambelli, T.; Vorholt, J. A.; Poulikakos, D.; Ferrari, A. Toward a rational design of surface textures promoting endothelialization. Nano Lett. 2014, 14 (2), 1069-79.

(46) Potthoff, E.; Guillaume-Gentil, O.; Ossola, D.; Polesel-Maris, J.; LeibundGut-Landmann, S.; Zambelli, T.; Vorholt, J. A. Rapid and serial quantification of adhesion forces of yeast and Mammalian cells. PLoS One 2012, 7 (12), No. e52712.

(47) Meister, A.; Gabi, M.; Behr, P.; Studer, P.; Voros, J.; Niedermann, P.; Bitterli, J.; Polesel-Maris, J.; Liley, M.; Heinzelmann, H.; Zambelli, T. FluidFM: combining atomic force microscopy and nanofluidics in a universal liquid delivery system for single cell applications and beyond. Nano Lett. 2009, 9 (6), 2501-7. (48) Franco, D.; Klingauf, M.; Bednarzik, M.; Cecchini, M.; Kurtcuoglu, V.; Gobrecht, J.; Poulikakos, D.; Ferrari, A. Control of initial endothelial spreading by topographic activation of focal adhesion kinase. Soft Matter 2011, 7 (16), 7313-7324.

(49) Limpert, E.; Stahel, W. A.; Abbt, M. Log-normal distributions across the sciences: Keys and clues. BioScience 2001, 51 (5), 341352.

(50) Panagiotakopoulou, M.; Lendenmann, T.; Pramotton, F. M.; Giampietro, C.; Stefopoulos, G.; Poulikakos, D.; Ferrari, A. Cell cycledependent force transmission in cancer cells. Mol. Biol. Cell 2018, 29 (21), 2528-2539.

(51) Pasapera, A. M.; Schneider, I. C.; Rericha, E.; Schlaepfer, D. D.; Waterman, C. M. Myosin II activity regulates vinculin recruitment to focal adhesions through FAK-mediated paxillin phosphorylation. J. Cell Biol. 2010, 188 (6), 877-90.

(52) Bergert, M.; Lendenmann, T.; Zundel, M.; Ehret, A. E.; Panozzo, D.; Richner, P.; Kim, D. K.; Kress, S. J.; Norris, D. J.; Sorkine-Hornung, O.; Mazza, E.; Poulikakos, D.; Ferrari, A. Confocal reference free traction force microscopy. Nat. Commun. 2016, 7, 12814

(53) Lendenmann, T.; Schneider, T.; Dumas, J.; Tarini, M.; Giampietro, C.; Bajpai, A.; Chen, W.; Gerber, J.; Poulikakos, D.; Ferrari, A.; Panozzo, D. Cellogram: On-the-Fly Traction Force Microscopy. Nano Lett. 2019, 19 (10), 6742-6750.

(54) van Hoorn, H.; Harkes, R.; Spiesz, E. M.; Storm, C.; van Noort, D.; Ladoux, B.; Schmidt, T. The nanoscale architecture of forcebearing focal adhesions. Nano Lett. 2014, 14 (8), 4257-62.

(55) Stubb, A.; Laine, R. F.; Miihkinen, M.; Hamidi, H.; Guzman, C.; Henriques, R.; Jacquemet, G.; Ivaska, J. Fluctuation-Based SuperResolution Traction Force Microscopy. Nano Lett. 2020, 20 (4), $2230-2245$.

(56) Hansson, G. K. Inflammation, atherosclerosis, and coronary artery disease. N. Engl. J. Med. 2005, 352 (16), 1685-95.

(57) Erusalimsky, J. D.; Kurz, D. J. Cellular senescence in vivo: its relevance in ageing and cardiovascular disease. Exp. Gerontol. 2005, 40 (8-9), 634-42.

(58) Tzima, E.; del Pozo, M. A.; Shattil, S. J.; Chien, S.; Schwartz, M. A. Activation of integrins in endothelial cells by fluid shear stress mediates Rho-dependent cytoskeletal alignment. EMBO J. 2001, 20 (17), 4639-47. 
(59) Tzima, E.; Irani-Tehrani, M.; Kiosses, W. B.; Dejana, E.; Schultz, D. A.; Engelhardt, B.; Cao, G.; DeLisser, H.; Schwartz, M. A. A mechanosensory complex that mediates the endothelial cell response to fluid shear stress. Nature 2005, 437 (7057), 426-31.

(60) Cheung, T. M.; Yan, J. B.; Fu, J. J.; Huang, J.; Yuan, F.; Truskey, G. A. Endothelial Cell Senescence Increases Traction Forces due to Age-Associated Changes in the Glycocalyx and SIRT1. Cell. Mol. Bioeng. 2015, 8 (1), 63-75.

(61) Kar, B.; Delgado, R. M.; Frazier, O. H.; Gregoric, I. D.; Harting, M. T.; Wadia, Y.; Myers, T. J.; Moser, R. D.; Freund, J. The effect of LVAD aortic outflow-graft placement on hemodynamics and flow: Implantation technique and computer flow modeling. Tex Heart Inst J. 2005, 32 (3), 294-298.

(62) Rikhtegar, F.; Wyss, C.; Stok, K. S.; Poulikakos, D.; Muller, R.; Kurtcuoglu, V. Hemodynamics in coronary arteries with overlapping stents. J. Biomech 2014, 47 (2), 505-11.

(63) Bachmann, B. J.; Bernardi, L.; Loosli, C.; Marschewski, J.; Perrini, M.; Ehrbar, M.; Ermanni, P.; Poulikakos, D.; Ferrari, A.; Mazza, E. A Novel Bioreactor System for the Assessment of Endothelialization on Deformable Surfaces. Sci. Rep 2016, 6, 38861.

(64) Bachmann, B. J.; Giampietro, C.; Bayram, A.; Stefopoulos, G.; Michos, C.; Graeber, G.; Falk, M. V.; Poulikakos, D.; Ferrari, A. Honeycomb-structured metasurfaces for the adaptive nesting of endothelial cells under hemodynamic loads. Biomater. Sci. 2018, 6 (10), 2726-2737.

(65) Robotti, F.; Franco, D.; Banninger, L.; Wyler, J.; Starck, C. T.; Falk, V.; Poulikakos, D.; Ferrari, A. The influence of surface microstructure on endothelialization under supraphysiological wall shear stress. Biomaterials 2014, 35 (30), 8479-86. 